\title{
Prevalence of Staphylococcus aureus nasal carriers and antibiotic resistance among staff of clinical wards in Nikuee Hospital, Qom, Iran, in 2012
}

\author{
Zia Sheikholeslami N, MD ${ }^{1}$, Rezaeian M, $\mathrm{PhD}^{2}$, Heidarpoor A, $\mathrm{MSc}^{3}$, Hadavi M, MSc ${ }^{4}$, Tashakori M, \\ $\mathrm{PhD}^{5 *}$ \\ 1- Associated Prof., of Infectious Diseases, Dept. of Infectious Diseases, Qom University of Medical Sciences, Qom, Iran. \\ 2- Professor of Epidemiology and Statics, Faculty of Medicine, Dept. of Epidemiology and Biostatistics, Rafsanjan \\ University of Medical Sciences, Rafsanjan, Iran. 3- MSc in Microbiology, Dept. of Microbiology, Qom University of \\ Medical Sciences, Qom, Iran. 4- PhD by Research Student, Dept. of Anesthesiology, Rafsanjan Paramedical School, \\ Rafsanjan University of Medical Sciences, Rafsanjan, Iran. 5- Assistant Prof., Dept. of Laboratory Medicine, Rafsanjan \\ Paramedical School, Rafsanjan University of Medical Sciences, Rafsanjan, Iran
}

\begin{abstract}
Received: January 2016, Accepted: May 2016

Background: Nosocomial infections are an important cause of hospital mortalities. Staphylococcus aureus (S. aureus) carriers among medical staff are an important source of nosocomial infections. The purpose of this study was to investigate the prevalence of the nasal carriers of $S$. aureus and antibiotic resistance in staff of Nikuee Hospital, Qom, Iran.

Materials and Methods: In this cross-sectional study, 152 health care workers (HCWs) were assessed to determine the frequency of nasal carriage of $S$. aureus, the rates of methicillin-resistant $S$. aureus (MRSA), and their antibiotic resistance profiles in Nikuee Hospital in 2012. Clinical samples were cultured and $S$. aureus was detected using conventional bacteriologic methods. Antimicrobial susceptibility patterns against penicillin, vancomycin, erythromycin, clindamycin, cefazolin, cotrimoxazole, and doxycycline were evaluated using the Kirby-Bauer disk diffusion technique. Methicillin resistance was confirmed by oxacillin disk diffusion test. Data were analyzed in SPSS software using chi-square test and independent two-sample t-test.

Results: Among the processed samples, 34.2\% illustrated nasal carriage of $S$. aureus, and among them, $23.5 \%$ were carriers of MRSA. None of the isolates were resistant to vancomycin. However, $96.1 \%, 33.3 \%, 27.5 \%, 19.6 \%, 9.8 \%$, and $15.7 \%$ resistance to penicillin, erythromycin, clindamycin, cefazolin, co-trimoxazole, and doxycycline were observed. There was no significant relationship between sex, age, and number of years of healthcare service and positive nasal carriage.

Conclusions: Data obtained in this study revealed high prevalence of nasal carriage of $S$. aureus (34.2\%) in HCWs at Nikuee Hospital. Therefore, it can be concluded that the role of the hospital staff in Nikuee Hospital as a source of staphylococcal infections is prominent. Thus, to prevent nosocomial infections, we recommend the identification and treatment of the carriers among the staff of other hospitals.
\end{abstract}

Keywords: Staphylococcus Aureus, Drug Resistance, Hospital Personnel, Iran.

\section{Introduction}

Staphylococcus aureus is an important human pathogen and frequent cause of both nosocomial and community acquired infections associated with high mortality and morbidity around the world $(1,2)$.

$S$. aureus have a tendency to colonize in various parts of healthy human bodies such as the nasopharyngeal area and skin (3). Previous investigations have shown that the anterior nasal region is the area in which these bacteria most commonly collect. It is a potent and

\footnotetext{
Corresponding author: Mahnaz Tashakori, Dept. of Laboratory Medicine, Rafsanjan Paramedical School, Rafsanjan University of Medical Sciences, Rafsanjan, Iran.

Email: m_tashakori44@yahoo.com
} 
increasing risk factor for subsequent staphylococcal infection in both hospitals and communities $(4,5)$.

Colonization with methicillin-resistant $S$. aureus (MRSA) has been revealed to increase the risk of MRSA infection both immediately and in long term carriers (6).

The colonization rate of $\mathrm{S}$. aureus on the nose in the general population is about $30 \%$, although this rate is higher in health care workers (HCWs) than the general population $(7,8)$. Understanding the nasal carriage rate among HCWs and their antimicrobial resistance profiles is necessary for infection control and appropriate antibiotic therapy.

The average rate of nasal carriers of $S$. aureus and MRSA among HCWs has been reported to be $23.7 \%$ and $4.6 \%$, respectively (9). The prevalence of nasal carriage of $S$. aureus among hospital personnel reported by several studies in Iran varied from $11 \%$ to $43 \%$ (1016). However, no data have been provided on HCWs of hospitals in Qom, Iran, until now.

According to several reports, carriers can be eliminated through simple treatments (17). Such treatments had a beneficial role in reducing nosocomial infections (7).

The identification of HCWs colonized with $S$. aureus combined with hand hygiene and other precautions have been shown to be effective in reducing the transmission and controlling the spread of staphylococcal infections. Thus, this investigation was undertaken to study the nasal carriage rate of $S$. aureus and MRSA among HCWs at a hospital and to determine the antibiotic resistance profile of the isolates.

\section{Material and Methods}

This cross-sectional study was conducted in Nikuee Hospital of Qom University of Medical Sciences, Qom, Iran, in 2012. In total, 152 of the $180 \mathrm{HCW}$ sere included in this investigation. HCWs with a history of upper respiratory tract infection, nasal medication use, antibiotic therapy, and recent nasal surgery were excluded from the study. Demographic data, including sex, age, number of years of health care sevice, history of upper respiratory infection, antibiotic use, and recent nose surgery, were collected through the completion of a questionnaire by each individual.

Samples were obtained using sterile swabs from deep tissue of anterior nares. The swabs were immediately placed in the transport media and transferred to the microbiology laboratory. The samples were cultured in 5\% sheep blood agar and incubated at $37^{\circ} \mathrm{C}$ for 48 hours.

$S$. aureus strains were identified according to colony morphology and biochemical activities, and using coagulase test (18). Susceptibility profiles to different antibiotics, including penicillin (10 IU), vancomycin (30 ug), erythromycin (15 ug), doxycycline (30 ug), clindamycin (2 ug), cefazolin (30 ug), and trimethoprim-sulfamethoxazole $(1.25 / 23.75 \mathrm{u})$ (HI-MEDIA, India), were evaluated with the guidelines of the Clinical and Laboratory Standards Institute (CLSI) using Kirby-Bauer disk diffusion method (19).Methicillinresistant isolates were determined through oxacillin disk diffusion test ( 1 ug) using Muller-Hinton agar plates inoculated with a suspension (equivalent to 0.5 McFarland standards) of the isolated $S$. aureus. After incubation of the plates at $35{ }^{\circ} \mathrm{C}$ for 24 hours, the inhibition zones were measured.

Interpretive criteria (in $\mathrm{mm}$ ) for oxacillin disk diffusion tests regarding $S$. aureus were 13 $\mathrm{mm}$ as susceptible, $11-12 \mathrm{~mm}$ as intermediate, and $10 \mathrm{~mm}$ as resistant (20). S. aureus ATCC 25923 was used as control strain.

After data collection, statistical analyses were performed in order to determine the frequency of subjects positive for nasal carriage of $S$. aureus. The positive and negative subjects were grouped according to age, sex, and year of health care service. The relationship between nasal carriers of $S$. aureus and sex, age, and year of health care service was evaluated using statistical methods, including chi-square test and independent two-sample ttest, in SPSS software (version 16, SPSS Inc., Chicago, IL, USA). In all analyses, $\mathrm{P}$ values of 
less than 0.05 were considered as statistically significant.

\section{Results}

A total of $152 \mathrm{HCWs}$, consisting of 101 men $(66.4 \%)$ and 51 women $(33.6 \%)$ with the age range of 23 to 56 years participated in this study. Descriptive data of studied cases are presented in table 1 . Out of all cases screened, $51(33.5 \%)$ individuals were identified as nasal carriers of $S$. aureus. On the other hand, 101 cases $(66.5 \%)$ with negative culture of nasal samples for $S$. aureus were classified as noncarriers. According to figure 1, 37 (36.63\%) of the 51 nasal carriers were men, and only 14 $(27.45 \%)$ were women.

Table 1: Descriptive data on carriers and non-carriers of Staphylococcus aureus among staff of clinical wards in Nikuee Hospital

\begin{tabular}{|c|c|c|c|c|}
\hline & \multicolumn{2}{|c|}{$\operatorname{Sex}[N(\%)]$} & \multirow{2}{*}{$\begin{array}{l}\text { Age (year) } \\
\text { Mean } \pm \text { SD }\end{array}$} & \multirow{2}{*}{$\begin{array}{c}\text { Work experience (year) } \\
\text { Mean } \pm \text { SD }\end{array}$} \\
\hline & Male & Female & & \\
\hline Carrier & $37(36.63)$ & $14(27.45)$ & $36.20 \pm 7.90$ & $11.75 \pm 7.74$ \\
\hline Non-carrier & $64(63.37)$ & $37(72.55)$ & $35.74 \pm 8.06$ & $11.53 \pm 7.74$ \\
\hline
\end{tabular}

Data analysis according to sex, and mean age and years of health service between carriers and non-carries revealed no statistically significant difference $(\mathrm{P}>0.05)$.

Among 51 S. aureus isolates, $12(23.5 \%)$ and 39 (76.5\%) were detected as MRSA and methicillin sensitive $S$. aureus (MSSA), respectively, using oxacillin disk diffusion test.
The resistance profile of isolated $S$. aureus to the tested antimicrobial agents is presented in table 2. No resistance was observed against vancomycin in the evaluated cases. Among the tested antibiotics, co-trimoxazole, cefazolin, and clindamycin were found to be the most effective against isolated strains.

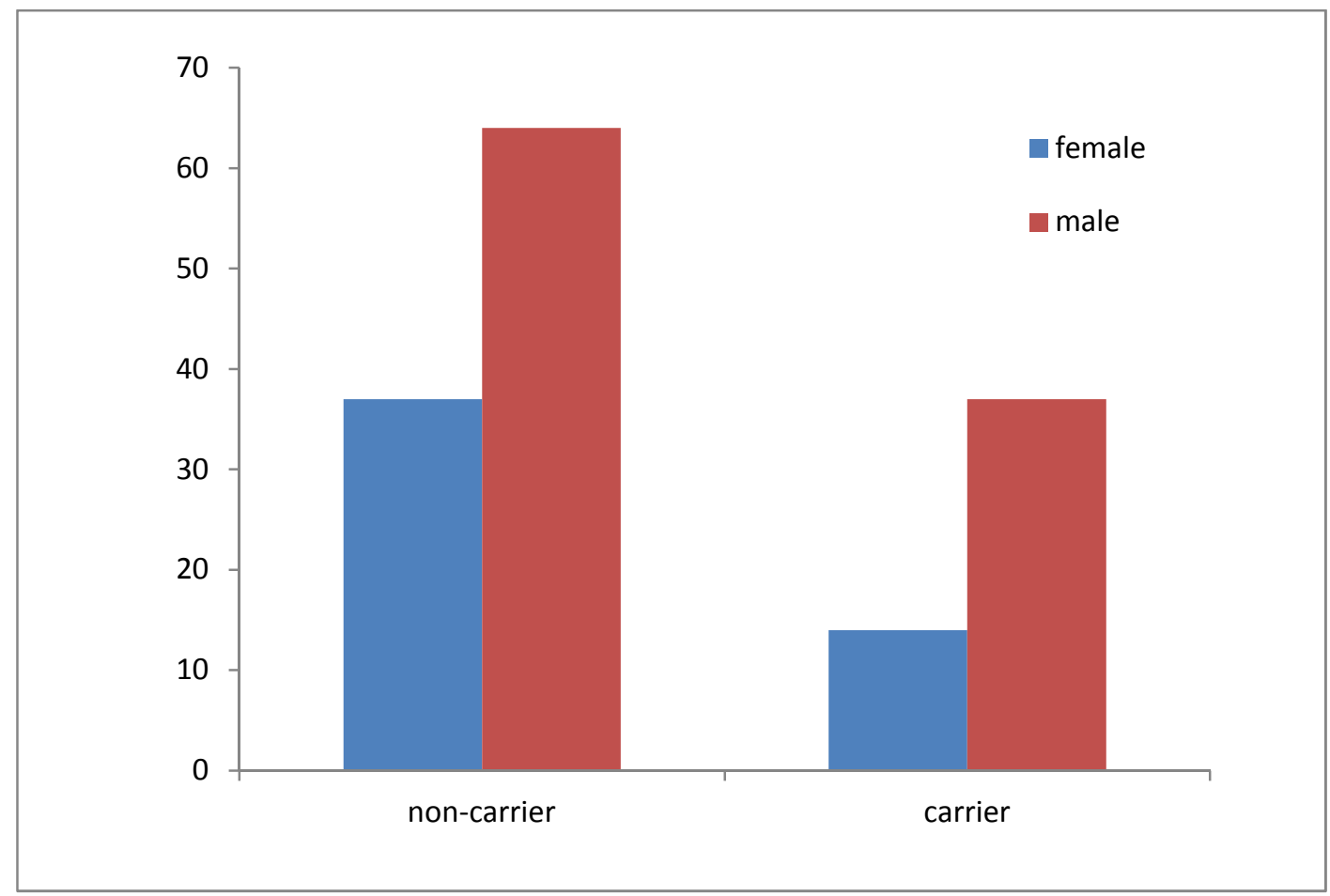

Figure 1: Distribution of carriage of Staphylococcus aureus in 151 health care workers in Nikuee Hospital according to gender 
Table 2: The antibiotic resistance patterns of isolated Staphylococcus aureus to the tested antimicrobial agents in health care workers at Nikuee Hospital

\begin{tabular}{llll}
\hline Antibiotics & $\begin{array}{l}\text { Resistance } \\
\text { Number }(\%)\end{array}$ & $\begin{array}{l}\text { Intermediate } \\
\text { Number }(\%)\end{array}$ & $\begin{array}{l}\text { Sensitive } \\
\text { Number }(\%)\end{array}$ \\
\hline Penicillin & $49(96.1)$ & $0(0.0)$ & $2(3.9)$ \\
\hline Doxycycline & $8(15.7)$ & $8(15.7)$ & $35(68.6)$ \\
\hline Co-trimoxazole & $5(9.8)$ & $0(0.0)$ & $46(90.2)$ \\
\hline Oxacillin & $12(23.5)$ & $0(0.0)$ & $39(76.5)$ \\
\hline Vancomycin & $0(0.0)$ & $0(0.0)$ & $51(100)$ \\
\hline Clindamycin & $14(27.5)$ & $1(2.0)$ & $36(70.5)$ \\
\hline Cefazolin & $10(19.6)$ & $1(2.0)$ & $40(78.4)$ \\
\hline Erythromycin & $17(33.3)$ & $6(11.8)$ & $28(54.9)$ \\
\hline
\end{tabular}

\section{Discussion}

HCWs are an important source of $S$. aureus and can host these organisms in the nasopharynx. Patients and hospital personnel colonized with $S$. aureus in hospitals or health care centers may transfer it to patients or their families through direct or close contact. In addition, they have a critical role in the epidemiology and pathogenesis of staphylococcal infections $(21,22)$. Nasal carriage of $S$. aureus varies between ethnicities, geographic regions, and different groups (23). There have been no reports on the prevalence of $S$. aureus nasal carriage among HCWs at Nikuee Hospital to date. In this investigation, a nasal carriage rate of $33.6 \%$ was observed among the studied cases. Of these, $23.5 \%$ were MRSA (i.e., $7.89 \%$ of all HCWs). Carriage of S. aureus in the nares has been detected in investigations in different countries including France (35.52\%), Northeast Ethiopia (28.8\%), Saudi Arabia (25.4\%), China (21.6\%), and Nepal (15.7\%) (24-28). In different studies in Iran, the frequency of nasal carriage of $S$. aureus in HCWs was reported between $11 \%$ in Bojnurd, Iran, and $43 \%$ in Sanandaj, Iran $(11,15)$. The prevalence of MRSA carriers ranged from 3\% to $30 \%$ in Iran, according to several reports (15). Data obtained in this study on the prevalence of $S$. aureus carries (33.6\%) was consistent with the results of the studies by Askari et al. in Namazi Hospital in Shiraz, Iran, and Rahbar et al. in Tehran, Iran, that reported a frequency of about $31 \%$ and $31.1 \%$, respectively $(10,13)$. The estimated frequency of nasal carriage in this study is higher than that reported by Moradi et al. (20.4\%), Sheikholeslami et al. (20\%), Ghafouri et al. $(11 \%)$, and Sharifi-mood et al. in Zahedan, Iran $(10 \%)(10,11,15-17)$. Nevertheless, our findings demonstrated lower prevalence of $S$. aureus carriers than the finding of Rashidian et al. in Sanandaj (43\%) (12). MRSA carriage rate $(7.89 \%)$ in the present study was consistent with the results of previous studies in Iran which reported a range of about $3 \%$ to $30 \%$ (15). The wide range of variation in nasal carriers can be attributed to differences in human population, sample size, study design, and sampling method.

In the present study, $100 \%$ susceptibility of isolates to vancomycin was observed. This finding was in agreement with that of previous reports in Iran $(10,14,17)$. However, vancomycin-resistant $S$. aureus have been reported in studies by Ghafouri et al., Ghasemian et al., and Saderi et al. in Iran (11, 14, 29). The highest resistance rate to penicillin was consistent with previous studies in Iran and other countries $(15,30,31)$. Low resistance rate of strains to co-trimoxazole was reported previously by Navidinia et al. (30). This was in agreement with the findings of the present study.

In this study, like several previous reports, no association was observed between $S$. aureus carriers and sex, age, and duration of health care service (15). However, Ohadian Moghadam et al. have reported a significant 
difference between sex and occupation with regard to MRSA carriage.

The major limitation of this study was that it was conducted only in one hospital in Qom. Further investigations which will help us to develop a better staphylococcal infections and spread of infection control are recommended. Thus, the evaluation of HCWs in different hospitals of Qom, assessment of the relation between nasal carriers of $S$. aureus among HCWs and work department, job type, and underlying diseases, and determination of mupirocin-resistant $S$. aureus among HCWs is suggested.

\section{Conclusion}

Since the HCWs have an important role as a reservoir for staphylococcal infection in hospital settings, regular screening and treatment of carriers among hospital staff is recommended in order to reduce transmission of staphylococcal infection in the hospital. Consequently, evaluation of antimicrobial resistance of staphylococcal isolates is necessary prior to antibiotic therapy in order to provide suitable treatment.

\section{Acknowledgement}

The authors appreciate the efforts of Maryam Bahsoun (Research Center, Ali-ibn Abi Talib Hospital, Rafsanjan, Iran) for her efforts in the translation of this paper and the laboratory personnel (Qom, Iran) for performing the laboratory tests.

Conflict of interests: None declared.

\section{References}

1. Bode LG, Kluytmans JA, Wertheim HF, Bogaers D, Vandenbroucke-Grauls CM, Roosendaal R, et al. Preventing surgical-site infections in nasal carriers of Staphylococcus aureus. New England Journal of Medicine. 2010;362(1):9-17.

2. Kluytmans J, Wertheim H. Nasal carriage of Staphylococcus aureus and prevention of nosocomial infections. Infection. 2005;33(1):38.

3. Kuehnert MJ, Kruszon-Moran D, Hill HA, McQuillan G, McAllister SK, Fosheim G, et al. Prevalence of Staphylococcus aureus nasal colonization in the United States, 2001-2002. Journal of Infectious Diseases. 2006;193(2):172-9.

4. Pérez-Vázquez M, Vindel A, Marcos C, Oteo J, Cuevas O, Trincado P, et al. Spread of invasive Spanish Staphylococcus aureus spatype 067 associated with a high prevalence of the aminoglycoside-modifying enzyme gene ant (4')-Ia and the efflux pump genes $\mathrm{msrA} / \mathrm{msrB}$. Journal of antimicrobial chemotherapy. 2009;63(1):21-31.

5. Wertheim HF, Vos MC, Ott A, van Belkum A, Voss A, Kluytmans JA, et al. Risk and outcome of nosocomial Staphylococcus aureus bacteraemia in nasal carriers versus noncarriers. The Lancet. 2004;364(9435):703-5.

6. Fard-Mousavi N, Mosayebi G, AmouzandehNobaveh A, Japouni-Nejad A, Ghaznavi-Rad E. The Dynamic of Staphylococcus aureus Nasal Carriage in Central Iran. Jundishapur journal of microbiology. 2015;8(7).

7. Saadatian-Elahi $M$, Tristan A, Laurent $F$, Rasigade J-P, Bouchiat C, Ranc A-G, et al. Basic rules of hygiene protect health care and lab workers from nasal colonization by Staphylococcus aureus: an international crosssectional study. PloS one. 2013;8(12):e82851.

8. Wertheim HF, Melles DC, Vos MC, van Leeuwen W, van Belkum A, Verbrugh HA, et al. The role of nasal carriage in Staphylococcus aureus infections. The Lancet infectious diseases. 2005;5(12):751-62.

9. Albrich WC, Harbarth S. Health-care workers: source, vector, or victim of MRSA? The Lancet infectious diseases. 2008;8(5):289-301.

10. Askarian M, Zeinalzadeh A, Japoni A, Alborzi A, Memish ZA. Prevalence of nasal carriage of methicillin-resistant Staphylococcus aureus and its antibiotic susceptibility pattern in healthcare workers at Namazi Hospital, Shiraz, Iran. International Journal of Infectious Diseases. 2009;13(5):e241-e7.

11. Ghafouri M, Besharati R, Lashkardoost H, Nojoomi S, Shakeri A, S. S. Prevalence of nasal carrier Staphylococcus aureus and their antibiotic resistance patterns among Health Care Working in Bojnurd Imam Reza Hospital. J North Khoras Univ Med Sci. 2014;6(1):1116.

12. Rashidian M, Taherpoor A, Goodarzi S. Nasal carrier rates and antibiotic resistance of Staphylococcus aureus isolates of Beasat Hospital staff. Scientific Journal of Kurdistan 
University of Medical Sciences 2001; 6(1):18.

13. Rahbar M, Karamiyar M, Gra-Agaji R. Nasal carriage of methicillin-resistant Staphylococcus aureus among healthcare workers of an Iranian hospital. Infection Control \& Hospital Epidemiology. 2003;24(04):236-7.

14. Saderi H, Oulia P, Jalali Nadoushan M, Falah N, Mohammadi Fallah F, Barati Namin M. The rate of staphylococcus aureus nasal carriage among personnels of a hospital in Tehran. Daneshvar Medicine 2004; 11(49):33-8.

15. Moradi-Tabriz H, Hadadi A, Sotoudeh-Anvari M, Rahimi-Foroushani A, Soleimani T, Mehdipour-Aghabagher B, et al. Prevalence and Antimicrobial Susceptibility of Staphylococcus aureus Isolated from Nasal Carriers. Journal of Medical Bacteriology. 2015;3(3-4):14-9.

16. Sharifi-Mood B, Metanat M, Alavi-Naini R, Shakeri A, Bameri Z, Imani M. Nasal carriage of methicillin-resistant staphylococcus aureus among ICU personnel working at Zahedan University, southeastern Iran. Caspian Journal of Internal Medicine. 2013;4(3):743.

17. Sheikholeslami N, Tashakori M, Bahsoun M. The Effectiveness of the Mupirocin Ointment in Treatment of Nasal Carriers of Staphylococcus aureus in Medical Staffs in Ali Ebne Abitaleb Hospital, Rafsanjan (Southeast of Iran). Trends in Medical Research. 2011;6:191-4.

18. Kateete DP, Kimani CN, Katabazi FA, Okeng A, Okee MS, Nanteza A, et al. Identification of Staphylococcus aureus: DNase and Mannitol salt agar improve the efficiency of the tube coagulase test. Annals of clinical microbiology and antimicrobials. 2010;9(1):1.

19. germany. Clinical and Laboratory Standards Institute. Performance standards for antimicrobial disk susceptibility tests. Approved standard M2-A10. Wayne, PA: Clinical and Laboratory Standards Institute; 2009.

20. Mimica M, Berezin E, Carvalho R, Mimica I, Mimica L, Sáfadi M, et al. Detection of methicillin resistance in Staphylococcus aureus isolated from pediatric patients: is the cefoxitin disk diffusion test accurate enough? Brazilian Journal of Infectious Diseases. 2007;11(4):4157.

21. Kakhandki LS, Peerapur B. Study of nasal carriage of MRSA among the clinical staff and health care workers of a teaching hospital of Karnataka, India. Al Ameen J Med Sci. 2012;5(4):367-70.
22. Sharma N, Singh S, Bains A. Phenotypic and genotypic characterization of MDR isolates of Staphylococcus aureus from urine and sputum in Himachal Pradesh. Drug Invention Today. 2012;4(10):497-500.

23. Ruimy R, Angebault C, Djossou F, Dupont C, Epelboin L, Jarraud S, et al. Are host genetics the predominant determinant of persistent nasal Staphylococcus aureus carriage in humans? Journal of Infectious Diseases. 2010;202(6):924-34.

24. Boisseau D, Alfandari S, Gauzit R, Rabaud C, Stahl J-P. Staphylococcus aureus nasal carriage during the infectious diseases national congress in France. Médecine et maladies infectieuses. 2012;42(9):435-9.

25. Shibabaw A, Abebe $T$, Mihret A. Nasal carriage rate of methicillin resistant Staphylococcus aureus among Dessie Referral Hospital health care workers; Dessie, Northeast Ethiopia. Antimicrob Resist Infect Control. 2013;2(1):25.

26. Alghaithy A, Bilal N, Gedebou M, Weily A. Nasal carriage and antibiotic resistance of Staphylococcus aureus isolates from hospital and non-hospital personnel in Abha, Saudi Arabia. Transactions of the Royal Society of Tropical Medicine and Hygiene. 2000;94(5):504-7.

27. Chen B, Dai X, He B, Pan K, Li H, Liu X, et al. Differences in Staphylococcus aureus nasal carriage and molecular characteristics among community residents and healthcare workers at Sun Yat-Sen University, Guangzhou, Southern China. BMC infectious diseases. 2015;15(1):1.

28. Khanal R, Sah P, Lamichhane P, Lamsal A, Upadhaya S, Pahwa VK. Nasal carriage of methicillin resistant Staphylococcus aureus among health care workers at a tertiary care hospital in Western Nepal. Antimicrobial resistance and infection control. 2015;4(1):1.

29. NAJAFI N, SHOJAI A, GHASEMIAN R. Nasal carriage and antibiotic resistance of Staphylococcus aureus isolates of Razi hospital personel, Qaemshahr, 1382. 2004.

30. Navidinia M. Detection of inducible clindamycin resistance (MLSBi) among methicillin-resistant Staphylococcus aureus (MRSA) isolated from health care providers. Journal of Paramedical Sciences. 2015;6(1).

31. Rioux C, Armand-Lefevre L, Guerinot W, Andremont A, Lucet J-C. Acquisition of methicillin-resistant Staphylococcus aureus in the acute care setting: incidence and risk factors. Infection Control \& Hospital Epidemiology. 2007;28(06):733-6. 